\title{
OBSERVABLE PROPERTIES OF NON-AXISYMMETRIC GALAXIES
}

\author{
II. Photometric Parameters
}

G. GALLETTA

Padova Astronomical Observatory, Padova, Italia

(Received 6 December, 1983)

\begin{abstract}
The analysis of triaxial, coaxial ellipsoids with different intrinsic axial ratios initiated in a previous paper is extended here, introducing an intrinsic distribution of light for each shell.

The general properties of this model are studied, considering the analytical solutions for the projection along a line-of-sight in three theoretical cases: (i) dust-free systems; (ii) dusty systems; (iii) luminous gaseous shells. The first case is then extended in order to predict some observable consequences.

The observed properties of both an exponential and a power-law luminosity profile are compared with those of triaxial and axisymmetric systems. In addition, the variation of the central surface brightness and of the isophotal flattening at a fixed level with respect to the inclination of the Galaxy are analyzed.
\end{abstract}

\section{Introduction}

In a previous paper (Galletta, 1983; hereafter referred to as Paper I) we studied a galaxy model in which the isodensity shells were represented by triaxial and coaxial ellipsoids of different intrinsic axial ratios. The above study was confined to the properties of the geometrical projection of the ellipsoidal shells; however, we showed that some observed effects like the twisting distribution with respect to the projected flattening (Galletta, 1980; Leach, 1981), or the presence of skewed dust lanes in some stellar systems (Hawarden et al., 1981) could also be expected for this theoretical galaxy population.

Here we would to extend the analysis of the general theory of ellipsoidal shells to a slightly higher degree of complexity, introducing an intrinsic luminosity density for each shell. Our aim is to see how the presence of triaxiality in the light distribution of a galaxy could influence its photometric properties like the projected luminosity profiles or the twisting.

We shall consider here that the matter and light isodensity surfaces coincide and are representable by $n$ ellipsoid equations. The projected density of light will then be computed assuming a function $I=I(n)$ describing the luminosity density within the system. The application of this result to some particular observable cases will be considered further on.

\section{Integrated Luminosity Density}

To investigate the properties of the projected luminosity generated by a generically triaxial system we consider a reference system $\hat{O X Y Z}$ with orthogonal axes centered on 
the Galaxy in which each isodensity shell can be represented by the equation

$$
x^{2} / a^{2}+y^{2} / b^{2}+z^{2} / c^{2}=1 .
$$

In this hypothesis the length $a$ represents the major axis of the shell, while $c$ represents the minor one.

We now define a new orthogonal reference system $\hat{O} X^{\prime} Y^{\prime} Z^{\prime}$ generated by two subsequent rotations of angles $\varphi$ and $\theta$ around the $Z$ and $Y^{\prime}$ axes, respectively. If we make the line-of-sight coincident with the $X^{\prime}$ axis, then the angles $\theta$ and $\varphi$ represent, respectively, the zenithal and the azimuthal angles of the observer's direction with respect to the major axis of the Galaxy. The plane $Y^{\prime} Z^{\prime}$ in this case will represent the plane of the sky. The equation describing each isodensity shell in this new system will be (see also Paper I)

$$
A x^{\prime 2}+B y^{\prime 2}+C z^{\prime 2}+D x^{\prime} y^{\prime}+E x^{\prime} z^{\prime}+F y^{\prime} z^{\prime}=1 \text {. }
$$

If we suppose that in each shell the mass-to-light ratio is constant along the surface, Equation (2) represents in the observer's reference system the surfaces of equal luminosity in space. Integrating the luminosity density of each shell along the $X^{\prime}$ direction we get the corresponding luminosity distribution on the plane of the sky. To obtain the luminosity projected on the plane of sky at each point $P^{*}=\left(y^{*}, z^{*}\right)$ we consider a line parallel to the $x^{\prime}$ axis and crossing the sky plane at $P^{*}$. This line crosses the $n$th shell at distances $x^{\prime}$ from the center satisfying the condition

$$
A_{n} x^{\prime 2}+\left(D_{n} y^{\prime *}+E_{n} z^{\prime *}\right) x^{\prime}+\left(B_{n} y^{\prime * 2}+C_{n} z^{\prime 2}+F_{n} y^{\prime} z^{\prime *}\right)=1,
$$

where the coefficients $A_{n}, \ldots, F_{n}$ refer to the $n$th shell. Solving Equation (3) we get

$$
x^{\prime}=-P_{n} / 2 S_{n}+\sqrt{\left(P_{n}^{2} / 4-S_{n} Q_{n}\right)} / S_{n},
$$

where we have set

$$
\begin{aligned}
& P_{n}=D_{n} y^{\prime}+E_{n} z^{\prime *}, \\
& S_{n}=A_{n}, \\
& Q_{n}=B_{n} y^{\prime} * 2+C_{n} z^{\prime}{ }^{2}+F_{n} y^{\prime} z^{\prime} *-1 .
\end{aligned}
$$

The coefficients of the Equations (5) are functions of the main semi-axes $a_{n}, b_{n}, c_{n}$ of the $n$th shell and of the angles of sight $\theta$ and $\varphi$. Since we want to use the same formalism as that of Paper I, we shall express Equation (4) in terms of the coefficients

$$
\begin{aligned}
& k_{n}=\sin 2 \varphi \cos \theta\left(p_{n}^{2}-1\right), \\
& s_{n}=\cos ^{2} \varphi\left(p_{n}^{2}-1\right)+1, \\
& j_{n}=q_{n}^{2}+\cos ^{2} \theta\left(p_{n}^{2}-q_{n}^{2}+1-s_{n}\right), \\
& T_{n}=a_{n}^{2} T^{\prime}=a_{n}^{2}\left(q_{n}^{2} s_{n}+\cos ^{2} \theta\left(p_{n}^{2}-q_{n}^{2} s_{n}\right)\right) ;
\end{aligned}
$$

describing the geometrical projection of the $n$th shell onto the plane of the sky by means 
of the equation

$$
j_{n} y^{\prime 2}+k_{n} y^{\prime} z^{\prime}+s_{n} z^{\prime 2}=T_{n}
$$

The new form of Equation (4) is then, for $\theta \neq m \pi / 2(m=1,2, \ldots)$

$$
\begin{aligned}
x_{n}^{\prime}= & \left(\operatorname{tg} \theta\left(q_{n}^{2} k_{n} y^{\prime *} / 2+\left(q_{n}^{2} s_{n}-T_{n}^{\prime}\right) z^{\prime *}\right) \pm\right. \\
& \left. \pm q_{n} p_{n} \sqrt{a_{n}^{2} T_{n}^{\prime}-j_{n} y^{\prime * 2}-k_{n} y^{\prime *} z^{\prime *}-s_{n} z^{\prime *}}\right) / T_{n}^{\prime}
\end{aligned}
$$

while, if $\theta=m \pi / 2$,

$$
x_{n}^{\prime}=\left(\sin 2 \varphi q_{n}^{2}\left(p_{n}^{2}-1\right) / 2 \pm q_{n} p_{n} \sqrt{a_{n}^{2} T_{n}^{\prime}-j_{n} y^{\prime} *^{2}-k_{n} y^{\prime} * z^{\prime} *-s_{n} z^{\prime} *^{2}}\right) / T_{n}^{\prime} .
$$

Equations (8) and (9), together with (6) give us the values of $x^{\prime}$ with respect to the observer's reference system $\hat{O} X^{\prime} Y^{\prime} Z^{\prime}$ in which the line-of-sight crosses the $n$th isodensity shell. Both equations could be rewritten in the form

$$
x_{n}^{\prime}\left(y^{\prime *}, z^{*}\right)=M_{n}\left(y^{\prime *}, z^{*}\right) \pm N_{n}\left(y^{\prime}, z^{*}\right),
$$

where $M_{n}$ corresponds to the first part of Equations (8) or (9) according to whether $\theta \neq m \pi / 2$ or $\theta=m \pi / 2$. The two signs of $N_{n}$ represent the point between the center and the observer ('foreground intersection') and the point belonging to the opposite side of the Galaxy with respect to the observer ('background intersection'). The thickness of space between shells $n$ and $n-1$ is then

$$
\Delta x_{n, n-1}^{\prime}=\left(x_{n}^{\prime}-x_{n-1}^{\prime}\right)_{f}+\left(x_{n}^{\prime}-x_{n-1}^{\prime}\right)_{b},
$$

where the indices $f$ and $b$ refer to the foreground and the background intersections. According to the assumptions made, the light intensity $I$ emitted per unit volume $\mathrm{d} x^{\prime} \mathrm{d} y^{\prime} \mathrm{d} z^{\prime}$ by an isodensity shell will be constant over the surface of the shell. The intrinsic light distribution from the nucleus outwards would then be correctly described as a function $I(a)$ varying with distance from the center, parametrized by the semi-major axis $a$ of each shell. The projected light intensity $I_{p}$ at a point $P\left(y^{\prime}, z^{\prime}\right)$ in this case is expressed by the integral function

$$
I_{p}\left(y^{\prime}, z^{\prime}\right)=\int_{-\infty}^{+\infty} I\left[a\left(x^{\prime}, y^{\prime}, z^{*}\right)\right] \mathrm{d} x^{\prime} .
$$

We can parametrize Equation (11) in terms of $n$, the index corresponding to each one of the $N$ shells crossed by the line-of-sight, setting $n$ to increase towards. If in this approximation the $j$ th shell is the innermost shell tangent to the line-of-sight $\left(\Delta x_{j}^{\prime}=0\right)$ which contributes to the projected intensity $I_{p}$, we can approximate Equation (11) with the sum

$$
I_{p}\left(y^{\prime *}, z^{\prime *}\right)=\sum_{j+1}^{N} n I_{n} \Delta x_{n, n-1}^{\prime}\left(y^{\prime *}, z^{\prime *}\right) .
$$


Starting from the above equation, we can consider some cases for which it is possible to compute the luminosity profile: (i) dust-free systems; (ii) dusty galaxies; (iii) bright gaseous shells. In general, only the first case is of interest in the stellar system models and it will be analyzed in more detail.

\subsection{DUST-FREE STELLAR SYSTEMS}

The first case considered is that of a triaxial distribution of stars in which the contribution to the light projected along the line-of-sight comes from all sides of the Galaxy (Figure 1(a)). In other words, the system will be considered as a transparent set of

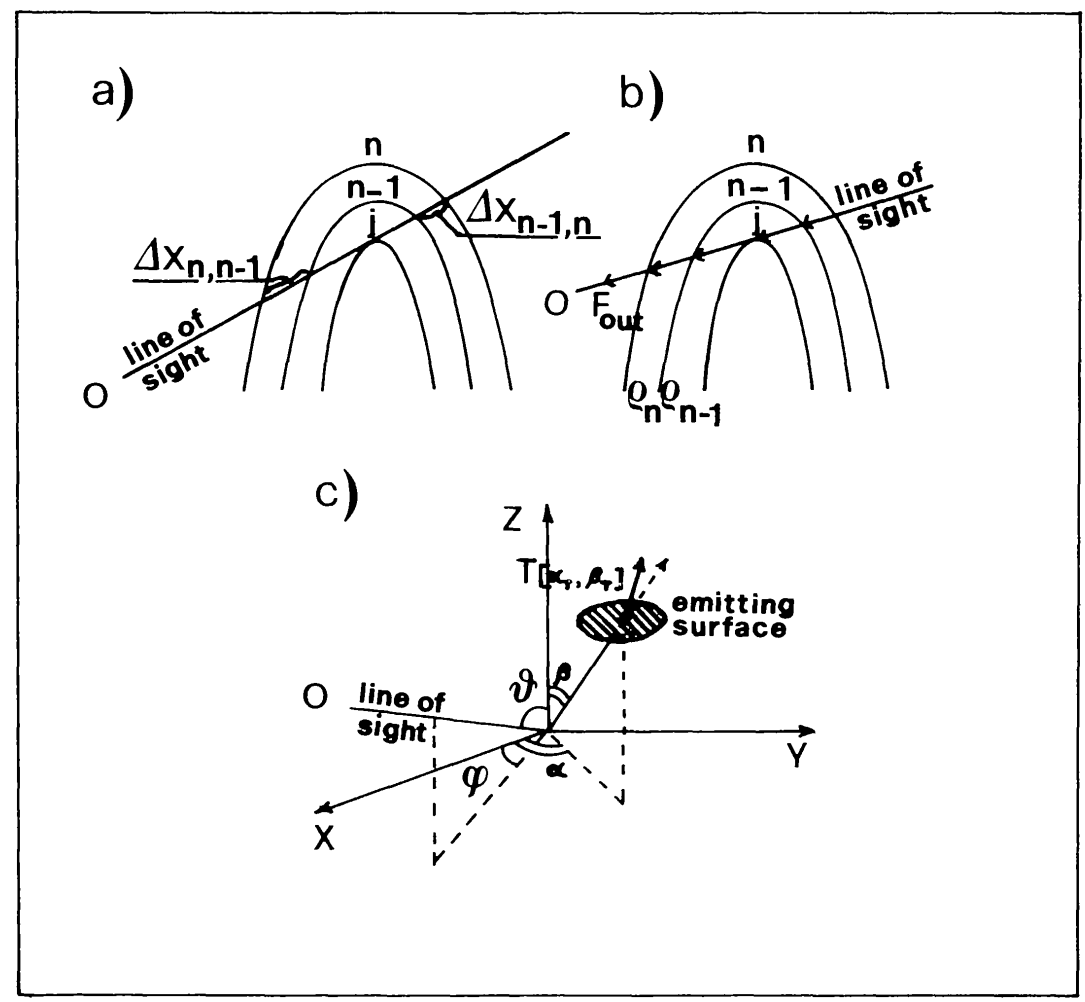

Fig. 1. (a) Section of the galaxy model showing the intersection of the line-of-sight with the $n$-shells. The thickness of each shell along the line-of-sight tangent to the $j$ th shell is also indicated. (b) The same as in (a), but in the case of absorbing matter, showing the attribution of the density $\rho_{n}$ to the corresponding region of space. (c) The relative orientation of the intrinsic reference system $X Y Z$ with the line-of-sight of the observer $O$. The normal to the emitting surface is indicated as $T\left(\alpha_{T}, \beta_{T}\right)$, while the zenithal and the azimuthal angles of the center of the surface are represented by the angles $\alpha$ and $\beta$ (see text).

ellipsoidal light emitting shells. In this case the thickness of shell $n$ (enclosed between the $n$th surface and the $(n-1)$ th one) could be written as

$$
\Delta x_{n, n-1}^{\prime}=\Delta x_{n, n}^{\prime}-\Delta x_{n-1, n-1}^{\prime}=2 N_{n}-2 N_{n-1},
$$

where $\Delta x_{n, n}^{\prime}=x_{n f}^{\prime}-x_{n b}^{\prime \prime}=M_{n}+N_{n}-\left(M_{n}-N_{n}\right)$. Setting $I_{N+1}=0$, where $I_{N+1}$ is the space luminosity outside the outermost shell, and remembering that $N_{j}=0$, we find that Equation (12) becomes

$$
I_{p}\left(y^{\prime} *, z^{\prime *}\right)=2 \sum_{j}^{N} N_{n}\left(I_{n}-I_{n+1}\right) \text {. }
$$


This latter equation will be used in the next section to compute the luminosity profile for different types of stellar systems.

Equations (11) or (14) could be used to generate a map of the Galaxy computed brightness by integrating the adimensional intensity (14) in small square cells of area $\mathrm{d} y^{\prime} \mathrm{d} z^{\prime}$; this would allow us to produce isophotal maps for the projected luminosity of the model. Actually, it is better from the analytic point of view to investigate how far the integrated isophote is from the geometrical projection of each shell, the analysis of which has been performed in Paper I. Along the projection of shell $j$, the coordinates $y^{\prime}, z^{\prime}$ on the plane of the sky are connected by means of Equation (7), giving for all these points $N_{j}=0$, while for the more external shells crossed by the line-of-sight we have

$$
z^{\prime *}=z^{*}\left(y^{*}, a_{j}, q_{j}, p_{j}, \theta, \varphi\right)
$$

and

$$
N_{n}=N\left(y^{*}, a_{n}, q_{n}, p_{n}, a_{j}, q_{j}, p_{j}, \theta, \varphi\right) .
$$

The resulting light intensity integrated along the line-of-sight for the points lying along the projection of the $j$ th shell is then given by

$$
I_{p}\left(y^{\prime}, z^{\prime}\right)_{j}=I_{j+1} \Delta x_{j+1, j}^{\prime}+\sum_{j+2}^{N}{ }_{n} I_{n} \Delta x_{n, n-1}^{\prime}=I_{r}+\sum_{j+2}^{N}{ }_{n} I_{n} \Delta x_{n, n-1}^{\prime} .
$$

Three cases can be considered:

(i) If $q$ and $p$ are slowly changing or constant with $n, \Delta x_{n, n-1}^{\prime} \simeq \Delta x$ is constant along the projection of the $j$ th shell, as well as $I_{p}\left(y^{\prime}, z^{\prime}\right)$.

(ii) If $I_{n} \gg I_{n+1}$, then dividing and multiplying $I_{p}$ by $I_{r}$, we obtain

$$
I_{p}\left(y^{\prime}, z^{\prime}\right)_{j}=I_{r}\left(1+\Delta x_{j+1, j}^{\prime} / I_{r}+\ldots\right) \simeq I_{r}=\text { const. }
$$

In both cases, the geometrical projections of the shells on the plane of the sky coincide with the isophotes observed when we integrate along the line-of-sight.

(iii) If, on the contrary, $q$ and $p$ are rapidly changing with respect to the luminosity decrease $\Delta I$, the shape of the isophotes does not correspond to the elliptical contours described by Equation (7).

In a single-component galaxy, the luminosity variation from one isophote to another is in most cases very steep, obeying an exponential or to an $r^{1 / 4}$-law (de Vaucouleurs, 1948, 1959; Freeman, 1970). The observed ellipticity on the other hand could either remains constant or change within a $\Delta\left(b_{0} / a_{0}\right) \simeq 0.5$, but this variation would always be slower than the one in $I_{p}$. This fact has been also noted by Williams (1981) in the application of a triaxial model to the $E$ galaxy NGC 596 and agrees with the above hypotheses (i) and (ii) making the geometrical projection of each shells a good approximation of their isophotes. Different conclusions must be reached, of course, if we are dealing with two component systems, in which the presence of lens or bars could generate sudden changes of ellipticity. 


\subsection{DUSTY SYSTEMS}

When absorbing matter is present in the Galaxy, it can no longer be considered as a transparent system and the integration along the line-of-sight takes a more complicate form. We could assume that each shell is similar to an absorbing and emitting layer whose absorption coefficient is $\chi$. The absorption of an incoming light intensity $F_{\text {in }}$ passing through a shell in which the absorbing material has a density $\rho$ will be proportional to the $\chi \rho \Delta x^{\prime}, \Delta x^{\prime}$ being the thickness of the shell. The total light intensity arising from that shell along the line-of-sight is then

$$
F=F_{\text {in }} \exp \left(-\chi \rho \Delta_{x}{ }^{\prime}\right)+I \Delta x^{\prime},
$$

where $x_{n}^{\prime}=M_{n}-N_{n}$ for $N_{n}>0$ represents the background intersection of the $n$th shell, and

$$
\Delta x_{n-1, n}=M_{n-1}-M_{n}-N_{n-1}+N_{n}
$$

is the corresponding thickness along $x^{\prime}$. By defining an absorption factor $\beta_{n}=\chi \rho_{n}$ for each shell (see Figure 1(b)), the total intensity of light arising from the $n$th shell for the background intersection along the line-of-sight will be given by

$$
F_{n b}=I_{n} \Delta x_{n-1, n}^{\prime}+F_{n+1, b} \exp \left(-\beta_{n} \Delta x_{n-1, n}^{\prime}\right) ;
$$

and for the foreground intersection,

$$
F_{n f}=I_{n} \Delta x_{n, n-1}^{\prime}+F_{n-1, F} \exp \left(-\beta_{n} \Delta x_{n, n-1}^{\prime}\right) .
$$

Applying $2 N$ times the above formulae (18) and (19) for both the background and the foreground intersections of each one of the $N$ shells along the line-of-sight, we find a final formula giving the flux $F_{N}\left(y^{\prime}, z^{\prime}\right)$ projected from a series of ellipsoidal shells containing absorbing and emitting material along the line-of-sight at a point $P\left(y^{\prime}, z^{\prime}\right)$ on the plane of the sky. To symmetrize the formula, we assume that $I_{N+1}=0$ and $\rho_{N+1}=0$, where $I_{N+1}, \rho_{N+1}$ are the luminous intensity and the density of absorbing matter outside the galaxy model, respectively. If the $j$ th shell is tangent to the line-of-sight, the light intensity received is then

$$
\begin{aligned}
F_{N}\left(y^{\prime}, z^{\prime}\right)= & \sum_{j+1}^{N}{ }_{k} I_{k} \Delta x_{k, k-1}^{\prime} E_{1}(k+1, N)+I_{j} \Delta x_{j, j}^{\prime} E_{1}(j+1, N)+ \\
& +\sum_{j+1}^{N}{ }_{k} I_{k} \Delta x_{k-1, k}^{\prime} E_{1}(j, N) E_{2}(j, k) ;
\end{aligned}
$$

or, in expanded form,

$$
\begin{aligned}
F_{N}\left(y^{\prime}, z^{\prime}\right)= & \sum_{j+1}^{N} I_{k}\left[\left(E_{1}(k+1, N)-E_{1}(j, N) E_{2}(j, k)\right)\left(M_{k}-M_{k-1}\right)+\right. \\
& \left.+\left(E_{1}(k+1, N)+E_{1}(j, N) E_{2}(j, k)\right)\left(N_{k}-N_{k-1}\right)\right]+ \\
& +2 I_{j} N_{j} E_{1}(j+1, N)
\end{aligned}
$$


where

$$
E_{1}(n, N)=\exp \left(-\sum_{n}^{N+1} \beta_{m} \Delta x_{m, m-1}^{\prime}\right)
$$

and

$$
E_{2}(n, k)=\exp \left(-\sum_{m}^{k-1}{ }_{m} \beta_{m} \Delta x_{m-1, m}^{\prime}\right)
$$

are also functions of $\left(x^{\prime}, y^{\prime}\right)$ and of the model's geometrical parameters. Equation (21) could be reduced to the previous case of transparent shells by setting $\chi=0$. In the previous computation we implicitly assumed that the thickness of each shell is so small that the self-absorption of the light emitted within a shell is negligeable. In this case, the intensity $I_{n}$ of the $n$th shell must be replaced by an equivalent intensity $I_{n}^{\prime}$ which is a function of the thickness $\Delta x_{n, n-1}^{\prime}$ and of the parameter $\beta_{n}$.

\subsection{LUMINOUS GASEOUS SHELLS}

As a third case, we consider that of an opaque shell embedded within the Galaxy. The luminous intensity of this shell must be added to that received from the outer foreground regions. In this case we must take into account the fact that the light received within a small area $\mathrm{d} y^{\prime} \mathrm{d} z^{\prime}$ on the sky does not depend on the thickness of the shell but on the inclination of the emitting surface with respect to the plane of the sky. In the previous case this was taken into account when the resulting $I_{p}\left(y^{\prime}, z^{\prime}\right)$ was integrated within the $\mathrm{d} y^{\prime} \mathrm{d} z^{\prime}$ area. The highly inclined portion of shells presented a higher thickness than that normal to the line-of-sight. In this case, however, only the inclination of the shell determines the relative intensity received. For this reason we have assumed that the opaque shell could be approximate by a number of infinitesimal plane surfaces of area $\mathrm{d} A=\mathrm{d} l_{1} \mathrm{~d} l_{2}$ tangent to the shell along their surface. In this case (see Figure $1(\mathrm{c})$ ), $T\left(x^{\prime}, y^{\prime}, z^{\prime}\right)$ being the point in which the line-of-sight crosses the shell with intrinsic axial ratios $q=c / a$ and $p=b / a$ and $\beta^{*}$ and $\alpha^{*}$ being the zenith and azimuthal angles of point $T$ in spherical coordinates with respect to the intrinsic reference system of the shell, is then easy to show that the zenith angle $\beta_{T}$ and the azimuthal angle $\alpha_{T}$ of the normal to the tangent plane passing through $T$ are defined by the equations

$$
\begin{aligned}
& \operatorname{tg} \alpha_{T}=p^{-2} \operatorname{tg} \alpha^{*} \quad\left(\text { for } \alpha^{*} z^{\prime} 90^{\circ}\right), \\
& \operatorname{tg} \beta_{T}=q^{-2} p^{-2} \operatorname{tg} \beta^{*} \sqrt{\cos ^{2} \alpha^{*} p^{4}+\sin ^{2} \alpha^{*}} ;
\end{aligned}
$$

which coincides with $\alpha_{T}=\alpha^{*}$ and $\beta_{T}=\beta^{*}$ only if $q=p=1-$ i.e., if the shell is spherical. The relation between the surface $\mathrm{d} A$ on the Galaxy shell and that on the plane of the sky $\mathrm{d} y^{\prime} \mathrm{d} z^{\prime}$ is

$$
\mathrm{d} A=\mathrm{d} y^{\prime} \mathrm{d} z^{\prime}\left[\cos \beta_{T} \cos \theta+\sin \beta_{T} \sin \theta \cos \left(\varphi-\alpha_{T}\right)\right]^{-1},
$$

which gives the surface intensity $S_{p}=I^{\prime} \mathrm{d} y^{\prime} \mathrm{d} z^{\prime}=I \mathrm{~d} A$ measured within $\mathrm{d} y^{\prime} \mathrm{d} z^{\prime}$.

As previously planned, we shall consider now some applications of case (a) to real galaxies, trying to deduce how such photometrical properties as the shape of the 
luminosity profile or the apparent diameter could be influenced by the presence of triaxial shells within the Galaxy.

\section{Observable Properties}

In order to connect the previous equations with observable parameters, we produced a number of simulations using computer models formed by shells of outwardly decreasing density and whose intrinsic axial ratios $q=c / a$ and $p=b / a$ are also functions of the distance from the center. As in Paper I, all the shells are coaxials and can be described by the following series of $N$ equations:

$$
\begin{array}{ll}
x^{2} / a_{n}^{2}+y^{2} / b_{n}^{2}+z^{2} / c_{n}^{2}=1, & q\left(a_{n}\right)=q_{0}+q *\left[a_{n}-a_{1}\right], \\
p\left(a_{n}\right)=p_{0}+p_{*} q\left(a_{n}\right), & I_{n}(x, y, z)=f\left(a_{n}\right) / f\left(a_{1}\right) ;
\end{array}
$$

where $a_{n}, b_{n}, c_{n}$ are the main semi-axes of the $n$th shell and $q_{0}, p_{0}, q_{*}, p_{*}$ are modeldependent parameters. The intrinsic light intensity emitted at point $P(x, y, z)$ lying in the region of space enclosed between shell $n$ and $n-1$ is a decreasing function of the distance from the center of each shell. In our models we considered for simplicity two cases only for the intrinsic flattening: (i) constant $q$ and $p\left(q_{*}=p_{*}=0\right)$; (ii) flattening linearly increasing with the radius (i.e., central shells are more spherical than the outer ones, setting $\left.q_{*}<0, p_{*}<0\right)$. But as we shall show, the main conclusions will be
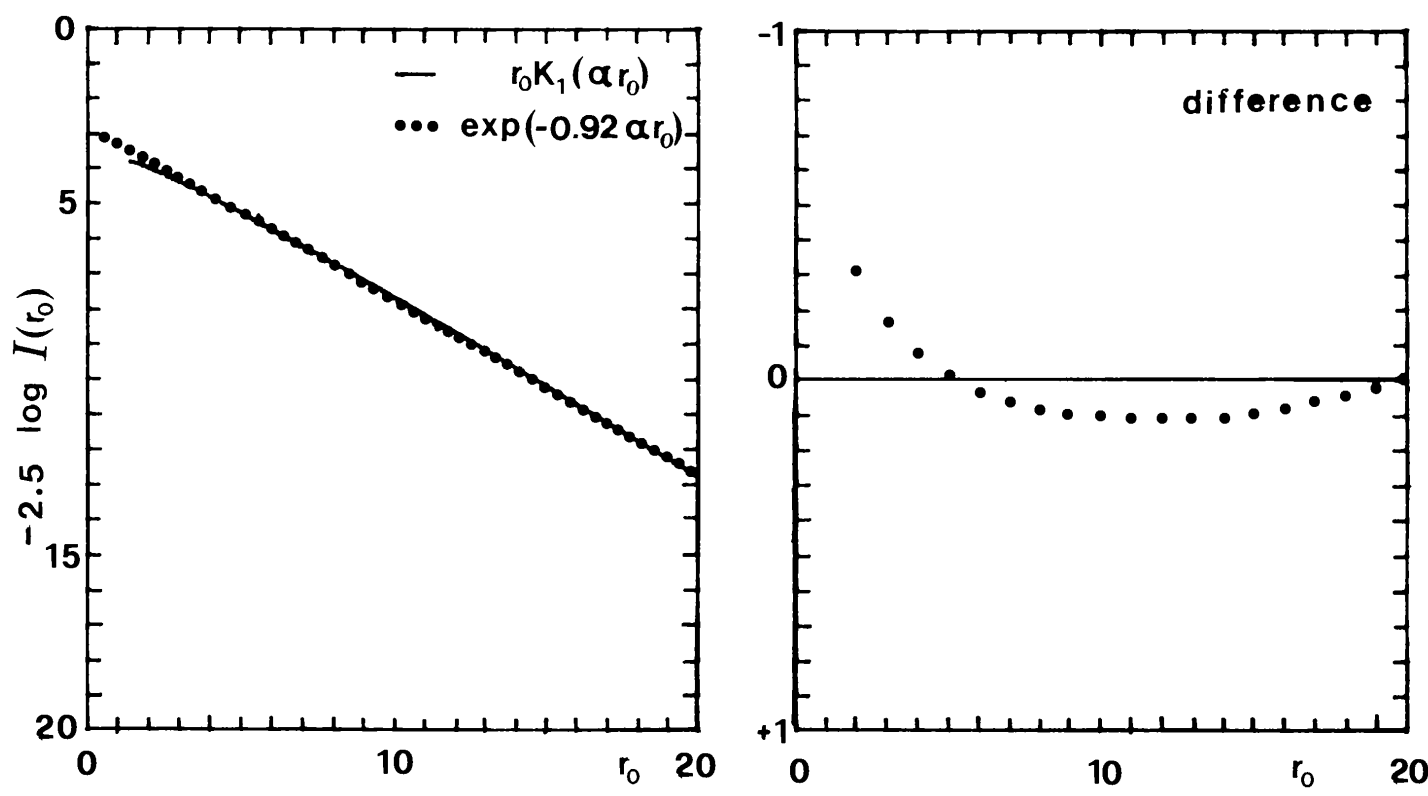

Fig. 2. Comparison of an exponential function $\exp \left(-0.92 \alpha_{i} r_{0}\right)$ with the function $r_{0} K_{1}\left(\alpha_{i} r_{0}\right)$ resulting from the projection of a function $\exp \left(-\alpha_{i} r\right)$ performed in an oblate galaxy seen edge-on. $K_{1}$ is a Bessel function of the second kind and of first order, which has been computed using a polynomial approximation truncated to $r_{0}^{-6}$. The zero point in the left diagram is arbitrary. The resulting differences in magnitudes per unit area are plotted in the right panel. With the exception of the inner part, the resulting difference is less than 0.2 mag. unit area. 
independent from the intrinsic flattening trends selected. As for the luminosity profiles, we focused our attention on two cases only: (i) Projected exponential law (Freeman, 1970), (ii) $R^{1 / 4}$-law (de Vaucouleurs, 1948).

Concerning the luminosity profile observed in disc galaxies (de Vaucouleurs, 1959; Freeman, 1970) we know that the integration along the line-of-sight of a pure exponen-
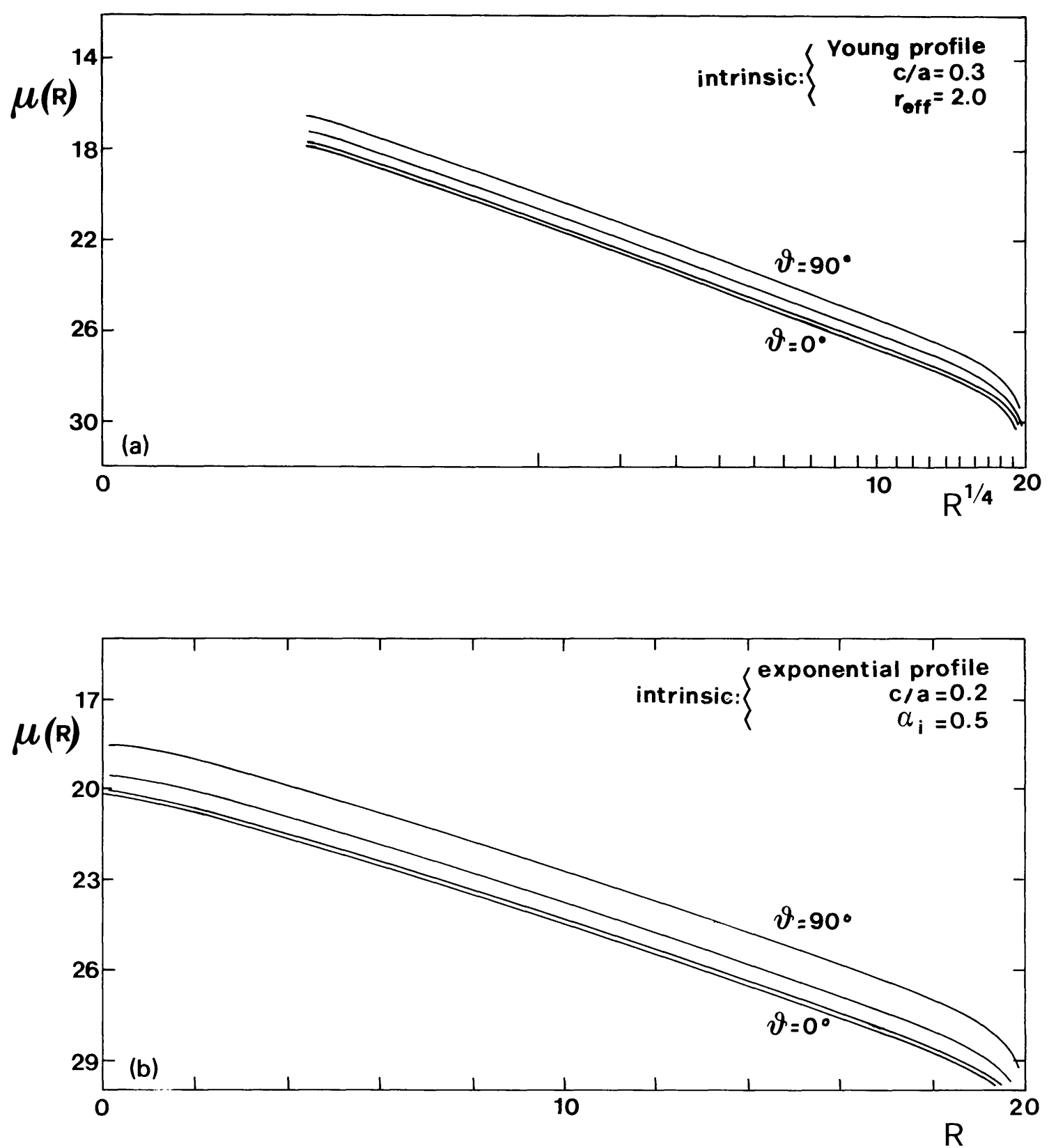

Fig. 3. Luminosity profiles computed along the apparent major axes of oblate models at different inclination angles. Panel (a) results from the projection of a Young luminosity profile (Young, 1976) with an effective radius of 2 length units within an oblate galaxy of constant flattenings $(q=0.3, p=1.0)$ extended 10 times its effective radius. Panel (b) represents the case of an exponential profile with slope 0.5 extended 20 length units. In this case the flattenings of the shells was everywhere set equal to $q=0.2, p=1.0$. In both cases $\theta$ represents the zenith angle (see Figure 1(c)). In these simulations the projected slope in the surface brightness versus $r^{1 / 4}$ (or $r$ ) diagrams is independent from the inclination of the Galaxy with respect to the plane of the sky. The zero-point of the $\mu(r)$ scale has been arbitrarly set. 
tial law $I(r)=\exp \left(-\alpha_{i} r\right)$ performed in an oblate galaxy seen edge-on produces as analytical solution a luminosity profile $I_{p}\left(r_{0}\right)=r_{0} K_{1}\left(\alpha_{i} r_{0}\right)$, with $K_{1}$ being a Bessel function of the second kind and of first order. When plotted in a $\log I-R$ diagram, this profile is very close to an exponential function in which the slope $\alpha_{p}$ is proportional to the intrinsic slope $\alpha_{i}$ by a factor $k$ slowly increasing with $\alpha_{i}$ and with a mean value of $\alpha_{p}=0.92 \alpha_{i}$ (see Figure 2). For this reason, we assumed in this paper that an intrinsic luminosity profile $\exp \left(-\alpha_{i} r\right)$ could represents fairly well the exponential profile observed in disc galaxies, providing that the intrinsic slope $\alpha_{i}$ be about 1.09 times the projected slope. This is also evident from Figure 3(b), where the luminosity profile obtained for an oblate model is plotted. In this figure the slope of the intrinsic (exponential) luminosity distribution has been assumed to be equal to 0.5 and the total radius equal to 20 length units. The magnitude-radius diagram obtained is linear for a large fraction of the extension of the Galaxy and span for an interval of about ten magnitudes. Larger total radii would have produced the same projected slope but with fainter external isophotes.

The $r^{1 / 4}$ law appear more complicate to define. A power law $I \propto 1 /\left(1+r^{-3.5}\right)$ gives projected profiles similar to the Hubble law but which deviate from the $r^{1 / 4}$ profile both in the inner that in the outer parts of the Galaxy. A better result has been obtained using the deprojected law estimated by Young (1976) in the asymptotic expansion for large $r$. The projection of the Young law is shown in Figure 3(a) using Equation (14).

These two laws have been then assumed as representative of the intrinsic luminosity distributions of the galaxy models and their projections on the sky have been parametrized by means of the relation

$$
\mu=-\gamma R^{n}+\delta
$$

where $\mu$ is the observed brightness and $\gamma$ and $\delta$ are parameters. For the exponential law we set $\gamma=2.5 \log e R_{0}^{-1}$ and $n=1, R_{0}=1 / \alpha_{p}$ being the scale-length, while the $R^{1 / 4}$ law is represented putting $n=\frac{1}{4}, R=R / R_{\text {eff }}$, and $\gamma=8.325$. In the last case $R_{\text {eff }}$ is the effective radius, which contains half of the total luminosity. In both cases $\delta=\mu(R=0)$ represents the central brightness assumed by the luminosity profile. The number of shells used in the simulations ranges from 100 for the exponential profiles to 500 for the Young profiles. These values have been assumed since in the first case the results are insensitive to an increase in the number of shells, while in the second case a central spike is produced by the innermost shell, as a consequence of the discrete approximation of the density distribution. The effects of the discreteness of the distribution can in fact be greatly reduced by increasing the number of shells present in the model, since this corresponds to reducing the distance between each shell and allows a better simulation of a smoothed distribution of stars.

The use of these models allow us to study some general features of the projected luminosity distributions which could be compared with the observed properties of real galaxies.

\subsection{CHANGE OF THE CENTRAL SURFACE BRIGHTNESS}

A first application of the models is represented by the study of the projected central 
brightness as a function of the angles $\theta$ and $\varphi$. Although in Equations (24) the intrinsic luminosity profile is defined in arbitrary units assuming a value of 1 for the first shell, when projected the resulting profile could be defined in mag. $\operatorname{arc~sec}^{-2}$ units, with the only uncertainty of its zero point. This latter could be defined, however, taking the peak brightness or by means of an inner extrapolation to $r=0$ of the main trend. The importance of this parameter in the photometry of the galaxies is stated by the fact that its observed value, when corrected to round isophotes by means of the factor

$$
\Delta \mu_{0}=2.5 \log \eta=2.5 \log \left(b_{0} / a_{0}\right)
$$

(Freeman, 1970), with $\eta$ being the apparent flattening, appear to be constant for systems obeying to the same luminosity law: about $21.65 \pm 0.3 \mathrm{mag} \mathrm{arc} \mathrm{sec}-2$ for disc galaxies (Freeman, 1970) and about $14.80 \pm 0.9 \mathrm{mag} \operatorname{arc~sec}^{-2}$ for spheroidal systems (Fish, 1964; Disney, 1976). If these type of systems are oblate, the deviation found for each galaxy from the 'face-on' corrected mean value could be due whether to observational errors or to a real difference in intrinsic brightness.

In our model the central brightness could be derived from the Equations (8), (9), (10), and (14) setting $x^{\prime}=y^{\prime}=0$. This gives

$$
I_{p}(0,0)=2 \sum_{j}^{N} q_{n} p_{n} a_{n} \sqrt{T_{n}^{\prime}\left(I_{n}-I_{n+1}\right)},
$$

which is function of $\theta$ and $\varphi$ and allows us to compute the value of the projected luminosity at the center of the system for each pair of angles. The above results could be analytically checked in the special case that the intrinsic flattenings $q$ and $p$ are constant. In fact, if the ellipsoidal shells could be represented by Equation (1), the intersections of the meridian plane containing the line-of-sight and inclined of an angle $\varphi$ with respect to the $X Z$-plane with the shells are ellipses of axial ratio $q^{\prime}=q \sqrt{\cos ^{2} \varphi+p^{-2} \sin ^{2} \varphi}$. The central intensity is then given by

$$
\begin{aligned}
I_{p}(\theta, \varphi, R=0) & =\int_{-\infty}^{+\infty} I(a) \mathrm{d} R(a, \theta, \varphi) \\
& =q /\left(\sin ^{2} \theta q^{\prime 2}+\cos ^{2} \theta\right)^{1 / 2} \int_{-\infty}^{+\infty} I(a) \mathrm{d} a,
\end{aligned}
$$

which is a function of $\theta$ and $\varphi$ only for a fixed pair of $q, p$ values and is independent of the intrinsic luminosity law. The brightness differences observed at the angles $\theta$ and $\varphi$ with respect to the $Z$-axis point of view (along the smaller axis) is the expressed by

$$
\begin{aligned}
\mu(\theta, \varphi, R=0)= & 1.25 \log \left[\cos ^{2} \theta+\sin ^{2} \theta q^{2}\left(\cos ^{2} \varphi+\sin ^{2} \varphi p^{-2}\right)\right]+ \\
& +\mu(0,0, R=0),
\end{aligned}
$$

which gives values equals to those computed using Equation (27). The change of central brightness with the orientation of the Galaxy in the interval $0^{\circ} \leq \theta \leq 90^{\circ}$, 
$-90^{\circ} \leq \varphi \leq 270^{\circ}$ is shown in Figure 4(a) and (b). In both cases we assumed $p=0.5$. The two plots are derived from the Equation (27) assuming a variable axial ratio $q$ (panel a) or a constant axial ratio of value 0.2 (panel b). The results obtained using different intrinsic ellipticity profiles and luminosity trends show that the central brightness changes with the angles-of-sight by a factor not greater than 1.8 mag., this figure being inversely dependent from the roundness of brighter shells.

The approximate constance of the central brightness for 'rounded' galaxies above discussed suggests an interesting test for the structure of the real systems. In fact, the rounding of the isotophotes corresponds to a face-on reduction in the case only of oblate systems. In this case $(p=1, q<1)$ the argument of the logarithm in Equation (29) is coincident with the apparent flattening of the Galaxy (Equation (21) of Paper I) and Equation (29) represents the constance of the face-on corrected central surface brightness. If the galaxies are prolate or triaxials $(p \neq 1)$, the face-on reduction of the central

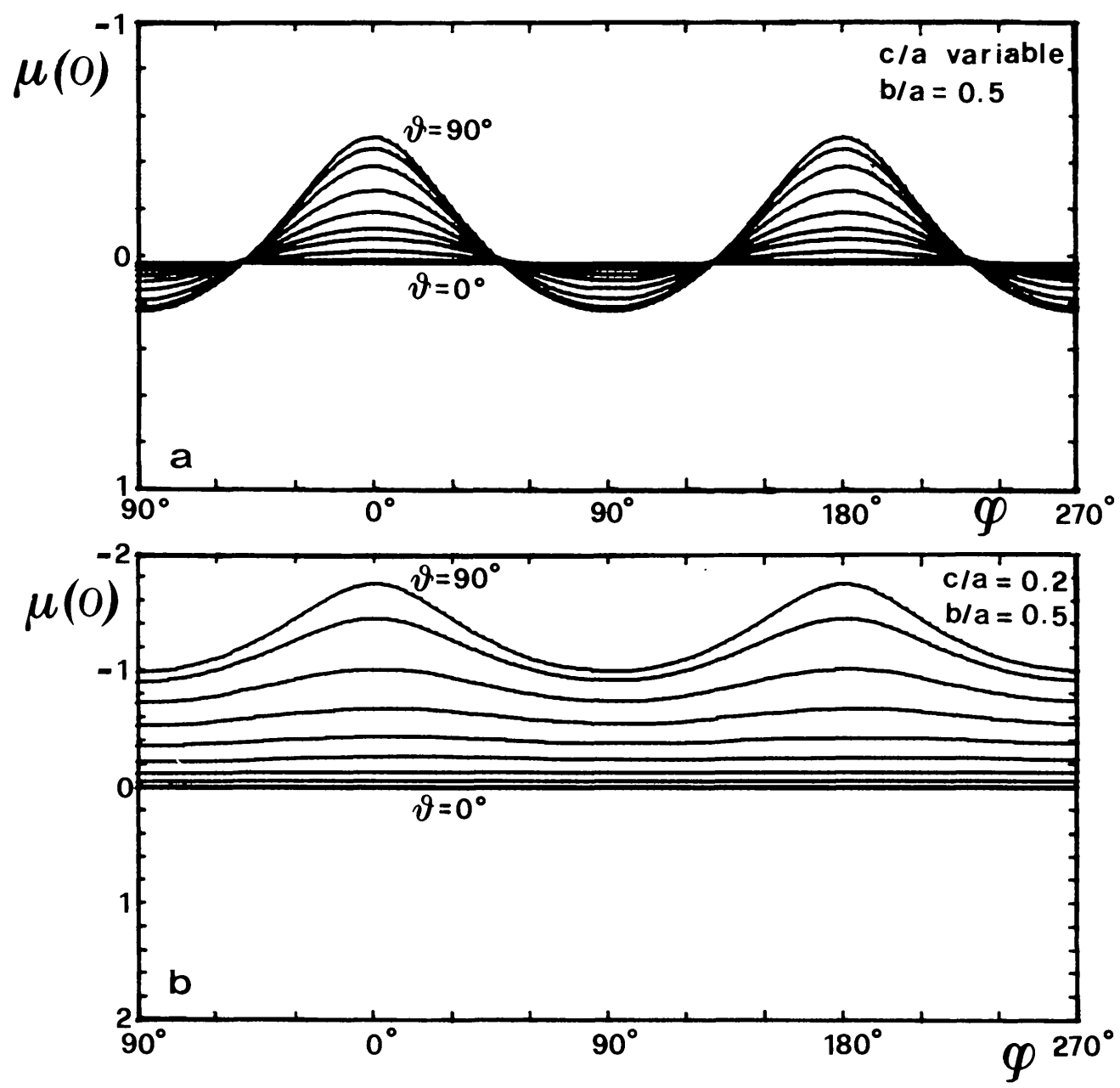

Fig. 4. Variation of the central brightness with respect to the position of the line-of-sight for triaxial galaxy models. In both cases we assumed for the axial ratio $p$ a constant value of 0.5 , studying the cases of an axial ratio $q$ constant and equal to 0.2 (panel (b)) or variable with the distance from the center from $q=0.9$ to $q=0.2$. 
brightness must be defined as $2.5 \log \left[\left(b_{0} a_{0}\right) /(b a)\right]$ which does not corresponds to the formula (26). In this hypothesis the application of the usual correction (26) to the observed values will increase the scatter of the single surface brightnesses around the mean value. Since the maximum scatter caused by the deviation from oblateness cannot be greater than the scatter of observed values, which includes observational errors and/or intrinsic differences between Galaxies, this poses an upper limit to the triaxiality or prolateness of the Galaxies. A comparison of Equations (29) applied to models possessing different intrinsic flattenings with the correction presented in Equation (26) shows that the axial ratio $b / a$ on the meridian plane (i.e., for triaxial systems, the plane containing the major and the intermediate axis) cannot be more flat than the limiting value $p^{*}$ defined by the relation

$$
-2.5 \log \left(p^{*}\right) \leq \sigma_{\mu},
$$

with $\sigma_{\mu}$ being the deviation found from the observations. This corresponds for disc galaxies $\left(\sigma_{\mu}=0.3\right)$ to an intrinsic flattening on the meridian plane not greater than 0.76 while for elliptical galaxies $\left(\sigma_{\mu}=0.9\right)$ their shape on the meridian plane could be as flatter as 0.44 .

\subsection{LUMINOSITY PROFILE}

Other interesting considerations could be drawn from the study of the luminosity profile variation with the radius at different angles of sight, applying Equation (14) in the logarithmic form

$$
\mu\left(y^{\prime}, z^{*}\right)=-2.5 \log \left(\sum_{j}^{N} N_{m}\left(I_{m}-I_{m+1}\right)\right)+\text { const. }
$$

Whichever of the two laws is used, we find that in an oblate system with constant flattening the slope $\gamma$ of the curve along the projected major axis in the brightness- $R^{n}$ diagram is independent from the particular value of flattening selected as well as from the inclination angle. The central brightness will be changing, however, generating a translation along the $\mu$-axis of the whole luminosity profile in the brightness- $R^{n}$ diagram. The global effects are shown in Figure 3 for two discs with constant flattening observed at different inclinations with respect to the equatorial plane and whose density distribution obeys to an exponential and a Young law, respectively. In the case of prolate systems with constant flattening, along the minor axis the slope is also independent from the inclination angles. In the more general case of triaxial galaxy with intrinsic flattenings changing with the distance from the center, the slope of the luminosity profile will be almost constant with the radius, except when the Galaxy appears close to its maximum flattening (edge-on vision plus minor axis profile), but its particular value will be dependent from the internal ellipticity trends as well from the inclination angles (Figure 5).

Concerning the slope projected along a line different from the apparent major axis, it is easy to show that, along the same isophote of apparent flattening $\eta_{0}=b_{0} / a_{0}$ (see Equation (21) of Paper I), we have $\gamma\left(\right.$ P.A.) $R^{n}=\gamma(0) a_{0}^{n}$, P.A. being the position angle of the luminosity profile computed assuming P.A. $=0^{\circ}$ along the apparent major axis 


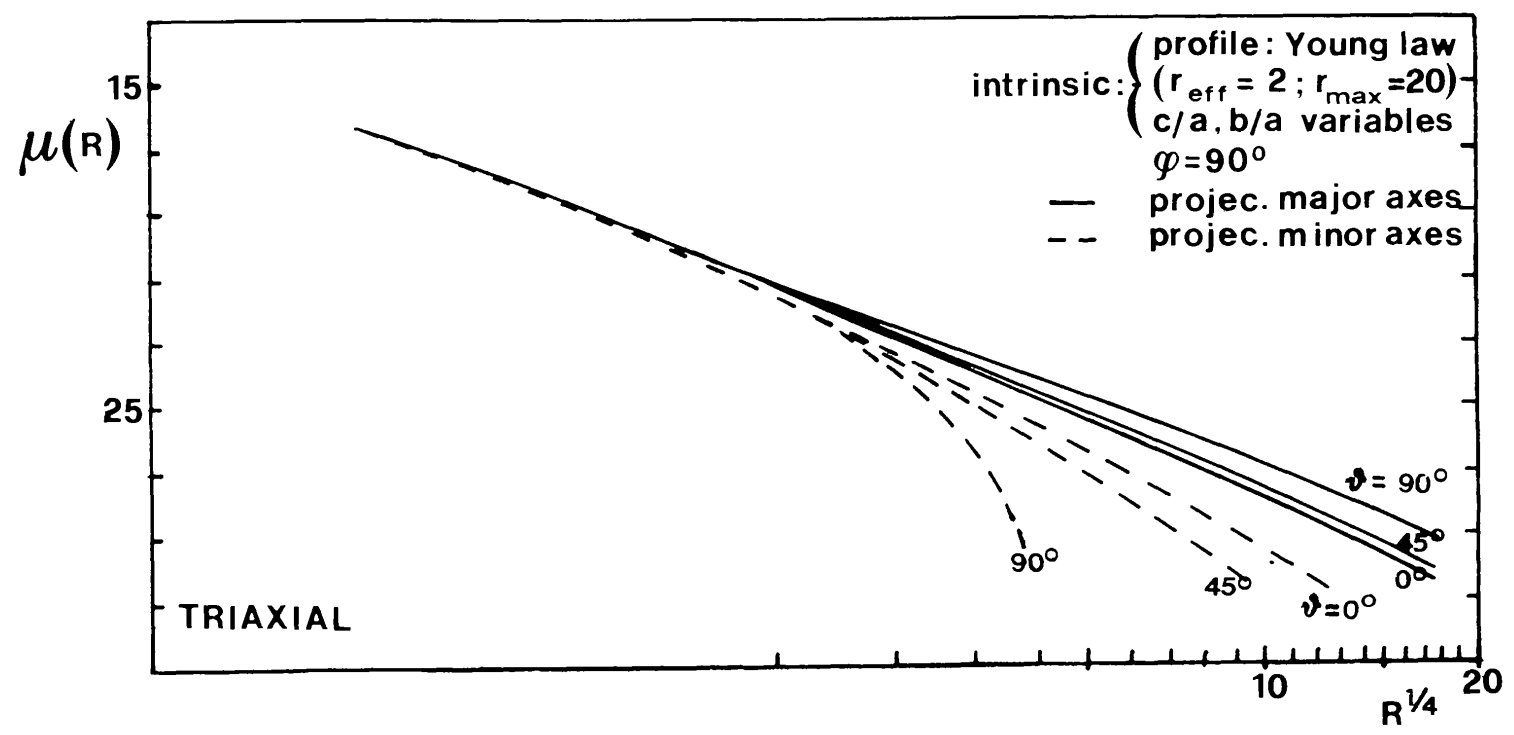

Fig. 5. Luminosity profiles computed for a triaxial galaxy model at different zenith angles and along the apparent major and minor axes. The intrinsic distribution used was a Young profile extended to 20 length units and with an effective radius equal to 2 length units. The axial ratios of the shells was variable from the center outward with values ranging from $q=0.9, p=0.98$ to $q=0.2, p=0.7$. The magnitude scale in the ordinate was plotted with an arbitrary zero point. Note as the deviation from the $r^{1 / 4}$ profile is noticeable only in edge-on vision $\left(\theta=90^{\circ}\right)$ and along the apparent minor axis.

$a_{0}$. Since the apparent isophote is an ellipse, the above relationship could be rewritten as

$$
\gamma(\text { P.A. })=\gamma(0)\left[\cos ^{2}(\text { P.A. })+\sin ^{2}(\text { P.A. }) \eta^{-2}\right]^{n / 2},
$$

which for an exponential profile $(n=1)$ along the apparent minor axis (P.A. $\left.=90^{\circ}\right)$ gives $\gamma(90)=\gamma(0) a_{0} / b_{0}$, in agreement with the model results.

\subsection{ApPARENT FLATTENING}

Another property belonging to models with changing intrinsic axial ratios with the distance from the center is the dependence of their apparent flattening at a fixed isophote in respect to the orientation angles and the intrinsic flattening trends. In the oblate case with constant axial ratios, the apparent flattening of the Galaxy on the sky is equal to $\eta=\sqrt{1-\sin ^{2} \theta\left(1-q^{2}\right)}$ independently of the surface brightness level selected. In a more general case, starting from Equation (31) computed for each point on the sky along the apparent major and minor axes it is possible to define for each level of surface brightness the corresponding isophotal flattening. When the intrinsic axial ratios change with the distance from the center, the relation between $\eta$ and $\theta$ deviates from the above formula, giving rounder or flatter isophotes according to the intrinsic trend of axial ratio (outwards increasing, decreasing, peaked, and so on).

Concerning the application of these results to real galaxies we compared the apparent flattenings exhibited at a previously selected brightness level $\mu^{*}$ by two different oblate models showing in an edge-on projection the same apparent flattening $\eta\left(\mu^{*}\right)$. The first 
one was assumed to have constant ellipticities; the second on the contrary is composed by shells with flattening changing from the center outwards. With the exception of models presenting sudden changes in the intrinsic ellipticity of the Galaxy, we found that the projected flattening corresponding to a fixed surface brightness level in the two above cases does not change very much with the inclination of the Galaxy in respect to the plane of the sky. This means that in a statistical study the apparent percentage of Galaxies observed at different apparent flattenings is not very much affected by the presence of a change of flattening within the Galaxy. Of course the exploration of all the combinations between luminosity profiles and ellipticity trends in statistical way is out of the purpose of this paper and the above result must be considered only as indicative. We note also that in all the simulations performed, when the flattening trends assumed in Equations (24) were smooth functions of the distance from the center, as is usually observed in the majority of elliptical galaxies, the isophotal flattening generated by each shell was very near to its geometrical projection on the sky.

\section{Conclusions}

The properties of galaxy models formed by shells of different axial ratios and possessing an intrinsic luminosity changing with the distance from the center have been investigated. Analytical solutions have been found for the case of transparent luminous shells as well as in the cases that dark or high density (and opaque) luminous matter also exists in the Galaxy. The main results of this study are:

(a) The central surface brightness obtained in the simulations of galaxies with exponential or $r^{1 / 4}$ luminosity profiles change with the orientation of the line-of-sight less than 2 mag. $\operatorname{arc~sec}^{-2}$, in agreement with the observations.

(b) The central surface brightness computed for each model, when corrected to round isophotes and compared with the observed values, could gives an upper limit for the deviation from oblateness eventually present in real galaxies. For disc systems, showing a central value $\mu_{0}=21.65 \pm 0.3 \mathrm{mag}$. arc $\sec ^{-2}$ (Freeman, 1970) the deviation from oblateness cannot be greater than an axial ratio in the meridian plane $p=0.76$. For ellipticals, with $\mu_{0}=14.80 \pm 0.9$ mag. $\operatorname{arc~sec}^{-2}$ (Fish, 1964; Disney, 1976), a triaxial shape is admissible until a value $p=0.44$.

(c) The slope of the projected luminosity law is almost constant with the radius. In addition, along the apparent major axis of oblate models and along the apparent minor axis of prolate ones, its value is independent from the inclination of the Galaxy with respect to the sky. The presence of triaxiality cannot influence the luminosity profile of a single galaxy, giving results similar to that of oblate case.

(d) A moderate change of the intrinsic flattening within a Galaxy possessing exponential or $r^{1 / 4}$ profile do not affects very much the isophotal flattening observed at a fixed surface brightness level. In addition, the flattening observed is in this case quite close to the geometrical projection of the single shells. 


\section{References}

Disney, M. J.: 1976, Nature 263, 573.

de Vaucouleurs, G.: 1948, Ann. Astrophys. 11, 247.

de Vaucouleurs, G.: 1959, in S. Flügge (ed.), Hdb. der Phys. 53, 511.

Freeman, K. C.: 1970, Astrophys. J. 160, 811.

Fish, R. A.: 1964, Astrophys. J. 139, 284.

Galletta, G.: 1980, Astron. Astrophys. 81, 179.

Galletta, G.: 1983, Astrophys. Space Sci. 92, 335 (Paper I).

Hawarden, T. G., Elson, R. A. W., Longmore, A. J., Tritton, S. B., and Corwin, H. G., Jr.: 1981, Monthly Notices Roy. Astron. Soc. 196, 747.

Leach, R. W.: 1981, Astrophys. J. 248, 485.

Young, P. J.: 1976, Astron. J. 81, 807.

Williams, T. B.: 1981, Astrophys. J. 244, 458. 